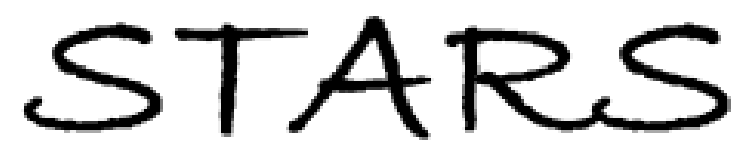

University of Central Florida

STARS

$1-1-2014$

\title{
High-performance near-infrared imaging for breast cancer detection
}

Yasser H. El-Sharkway

Ashraf F. El-Sherif

University of Central Florida

Find similar works at: https://stars.library.ucf.edu/facultybib2010

University of Central Florida Libraries http://library.ucf.edu

This Article is brought to you for free and open access by the Faculty Bibliography at STARS. It has been accepted for inclusion in Faculty Bibliography 2010 s by an authorized administrator of STARS. For more information, please contact STARS@ucf.edu.

\section{Recommended Citation}

El-Sharkway, Yasser H. and El-Sherif, Ashraf F., "High-performance near-infrared imaging for breast cancer detection" (2014). Faculty Bibliography 2010s. 5300.

https://stars.library.ucf.edu/facultybib2010/5300

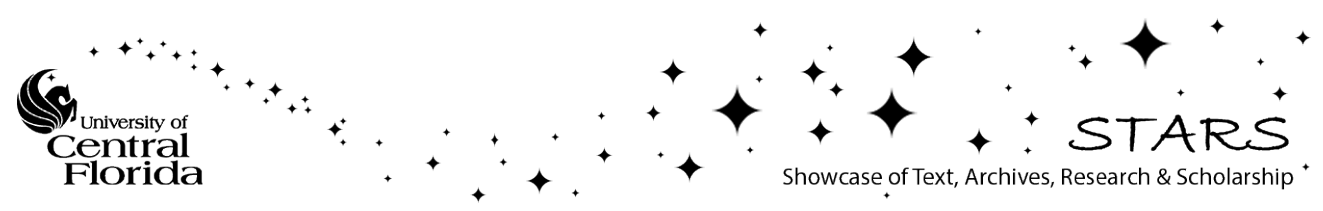




\section{Biomedical Optics}

\section{SPIEDigitalLibrary.org/jbo}

\section{High-performance near-infrared imaging for breast cancer detection}

Yasser H. El-Sharkawy

Ashraf F. El-Sherif 


\title{
High-performance near-infrared imaging for breast cancer detection
}

\author{
Yasser H. El-Sharkawy ${ }^{a}$ and Ashraf F. El-Sherif ${ }^{b, c, *}$ \\ ${ }^{a}$ Military Technical College, Department of Biomedical Engineering, Kobry Elkobbah, Cairo 11787, Egypt \\ ${ }^{b}$ Military Technical College, Department of Engineering Physics, Laser Photonics Research Group, Kobry Elkobbah, Cairo 11787, Egypt \\ ${ }^{\circ}$ CREOL, The College of Optics and Photonics, Building 53, 4000 Central Florida Boulevard, Orlando, Florida 32816
}

\begin{abstract}
We present a method for the noninvasive determination of the size, position, and optical properties of tumors in the human breast. The tumor is first detected by photothermal imaging. It is then sized, located, and optically characterized using designed digital image processing and edge-detection pattern recognition. The method assumes that the tumor is spherical and inhomogeneous and embedded in an otherwise homogeneous tissue. Heat energy is deposited in the tissue by absorption of near-infrared (NIR) Nd:YAG laser radiation, and its subsequent conversion to heat via vibrational relaxation causes a rise in temperature of the tissue. The tumor absorbs and scatters NIR light more strongly than the surrounding healthy tissue. Heat will diffuse through the tissue, causing a rise in temperature of the surrounding tissue. Differentiation between normal and cancerous tissues is determined using IR thermal imaging. Results are presented on a 55-year-old patient with a papillary breast cancer. We found that these results provide the clinician with more detailed information about breast lesions detected by photothermal imaging and thereby enhance its potential for specificity. ๑ 2014 Society of Photo-Optical Instrumentation Engineers (SPIE) [DOI: 10.1117/1.JBO.19.1.016018]
\end{abstract}

Keywords: near-infrared imaging; photothermal; edge-detection pattern; breast cancer detection.

Paper 130313RRRR received May 3, 2013; revised manuscript received Dec. 17, 2013; accepted for publication Dec. 30, 2013; published online Jan. 27, 2014.

\section{Introduction}

Photothermal imaging is a noninvasive imaging modality that employs near-infrared (NIR) light, typically in the wavelength range of $1064 \mathrm{~nm}$, to detect breast cancer. The basic approach consists of illuminating one side of the female breast samples with a pulsed light beam, while the examiner looks for regions of higher induced thermal intensity on the same side using a thermal camera.

Thermal effects are perhaps the most widely encountered form of tissue-laser interaction in clinical practice. Many such applications can be performed with long-pulse lasers and have been utilized clinically for nearly six decades. Understanding the physical mechanisms of laser interaction with biological molecules and structures has resulted in the application of laser radiation to a wide variety of biological and medical problems, ranging from subcellular manipulation/surgery to the successful diagnosis and treatment of human disease. ${ }^{1}$

At the tissue level, lasers are being used to diagnose and treat malignancy in combination with light-activated drugs, to ablate cornea and other hard and soft tissues through ultraviolet photoablation, to selectively ablate structures within the skin under controlled heating/cooling conditions, and to differentiate normal from abnormal tissue using a variety of fluorescence detection and light-scattering techniques. ${ }^{2,3}$

Thermal effects are important phenomena in laser interactions with biological tissues, resulting from three distinct phenomena; namely the conversion of light to heat, the transfer of heat and the tissue reactions, which are ultimately related to the change in temperature, and the heating time, as shown in the schematic diagram of Fig. $1 .^{4}$ A key for understanding

*Address all correspondence to: Ashraf F. El-Sherif, E-mail: ashraf.alsharif@ staff.aast.edu this interaction is through the analysis of the mechanisms of heat transfer in the biological tissues. This transfer is essentially produced by the mechanism of conduction, the influence of blood perfusion, and metabolic heat generation. ${ }^{4}$ The transfer of energy of the laser beam to the tissue molecules occurs randomly between the more and less energetic particles and results in a "secondary" heated volume; this is bigger than the "primary" laser source, which is based only on the conversion of light to heat. The kinetics of this thermal action, in turn, depend on the initial temperature in the tissue, time of heating, and susceptibility of the tissue to thermal damage or, in our case, thermal expansion. The lack of proper physical understanding of the process of laser-tissue interactions has been one of the principal deterrents in the full exploitation of the associated benefits in medical treatments. Over many years, medical cancer treatments using gold particles and procedures have remained as more or less an experience-based science than a subject of more fundamental study, ${ }^{5,6}$ but lately significant attempts are being made to understand the process of laser-tissue interaction from a fundamental view point. Fundamental mathematical studies with regard to laser-tissue photothermal interaction have been relatively few. ${ }^{7-12}$

\section{Theoretical Modeling}

Consider a laser beam incident on the tissue. When a steady state has been reached, the scattered incident light can be described by the irradiance, which depends on the position in the tissue $\psi=(x, y, z)$. The units of $\psi$ are $\mathrm{Wm}^{-2}$, so it is a measure of the energy flux through a unit area in a unit time. The transfer of irradiance to heat depends upon absorption and thermal 

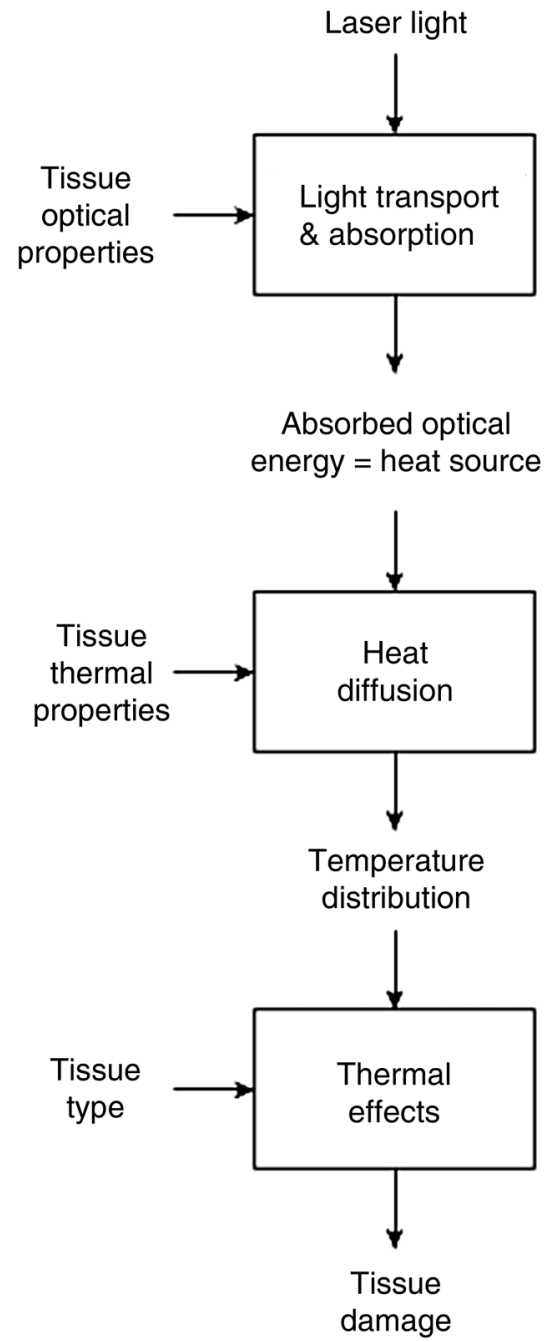

Fig. 1 The various aspects involved in thermal interactions of light with tissue.

relaxation, which leads to an increase in temperature as demonstrated in the next section.

\subsection{Temperature Rise}

If the tissue does not change the phase (our case study), then an increase in the heat energy per unit volume, $\partial E$, leads to a rise in temperature $\partial T$. The magnitude of this rise can be calculated using the specific heat capacity, $C_{p}{ }^{12-14}$

$C_{p} \cong \frac{1}{\rho}\left(\frac{\partial E}{\partial T}\right)_{p}$

A heat increase $\partial E$ (energy per unit volume) and the resulting temperature rise $\partial T$ are therefore related by

$\partial E=\rho C_{p} d T$

where $\rho$ is the mass density in $\mathrm{kgm}^{-3}$, and $C_{p}$ is the specific heat capacity in $\mathrm{Jkg}^{-1} \mathrm{~K}^{-1}$.

\subsection{Thermal Conductivity}

The deposited heat does not remain in the same place for a long time, but spreads into the surrounding tissue. We therefore need something to describe the flow of heat through the tissue. It is found empirically that the heat flux, $q$, is proportional to the gradient of the temperature, given by the vector

$\nabla T=\left(\frac{\partial T}{\partial x}, \frac{\partial T}{\partial y}, \frac{\partial T}{\partial z}\right)$

We can write that the heat flux and temperature gradient are related by

$q=-k \nabla T$

\subsection{Governing Equations}

The governing equation of thermal energy conservation in the tissues exposed to the laser irradiation is given by ${ }^{13,14}$

$$
\begin{aligned}
\rho C_{P} \frac{\partial T}{\partial t}= & k\left(\frac{\partial^{2} T}{\partial x^{2}}+\frac{\partial^{2} T}{\partial y^{2}}+\frac{\partial^{2} T}{\partial z^{2}}\right)+w_{b} C_{p b}\left(T_{a}-T\right) \\
& +Q_{m}-\frac{\partial \rho \Delta H}{\partial t}
\end{aligned}
$$

where $\rho, C_{p}$, and $k$ are, respectively, the density, the specific heat, and the thermal conductivity of the tissue, $Q_{m}$ is the metabolic heat generation per unit volume, $w_{b}$ is the blood perfusion rate, $C_{p b}$ is the specific heat of blood, $T_{a}$ is the initial temperature, and $T$ is the local tissue temperature.

The last term on the right side represents the rate of release/ absorption of latent heat, as a consequence of the phase change process; $\Delta H$ being the latent enthalpy characterizing phase transformations at the specific locations in the domain under consideration.

Solutions to simple cases (e.g., simple geometries and temperature-independent and uniform material properties) give us a guide to how heat behaves and can even be applied directly to a number of practical cases.

For convenience, we will define the heat source term as $\hat{Q} \equiv$ $Q / \rho C_{p}$ and introduce the thermal diffusivity $D\left(\mathrm{~cm}^{2} \mathrm{~s}^{-1}\right)$, defined by ${ }^{12-14}$

$D \equiv k / \rho C_{p}$

Equation (6) can then be written as

$\frac{\partial T}{\partial t}-D \nabla^{2} T=\hat{Q}(x, t)$

where the source has been explicitly written as a distributed source $\hat{Q}(x, t)$. The solution can now be calculated from the Green's function above by integrating (summing) together the Green's functions for every source position $x^{\prime}$ and time $t^{\prime}$ and weighted by the source term $\hat{Q}(x, t)$

$T(x, t)=\frac{E}{\rho c_{p}}\left[\frac{1}{8(\pi D t)^{3 / 2}}\right] \exp \left(-x^{2} / 4 D t\right)$.

At $1064 \mathrm{~nm}$, there was a clear and reproducible difference between the thermal response of cancerous and normal tissues as a result of increased optical attenuation in cancerous tissues, providing the capability to characterize abnormal tissue and give us information about both the composition and thickness of the tissue. 


\section{Experimental System}

Figure 2 shows a schematic drawing of the experimental setup. A Q-switched pulsed Nd:YAG laser with a wavelength of $1064 \mathrm{~nm}$, a pulse duration of $14 \mathrm{~ns}$, and a $10-\mathrm{Hz}$ repetition rate was used to illuminate the samples. To achieve a homogenous laser fluence distribution over the whole circular top surface of the sample, the laser beam was expanded by two concave lenses with -50 - and $-75-\mathrm{mm}$ focal lengths, respectively. Use of these lenses decreased the laser fluence incident on the biopsy sample surface to approximately $0.2 \mathrm{~mJ} \mathrm{~cm}^{-2}$, and this resulted in a weak signal-to-noise ratio. The black-body radiation from the optically excited sample is collected, collimated, and focused onto an IR thermal camera sensitive over 7.5 to $14 \mu \mathrm{m}$. The thermal camera (model Fluke Ti32 thermal imager) is coupled with computer software for the interpretation of the IR images. The test is ideal for detecting hot and cold spots or areas of different emissivities on the skin surface, since human tissue radiates IR energy very efficiently. ${ }^{15-19}$

\subsection{Image Acquisition}

To provide high-resolution and accurate photothermal images, the following procedures for data collections must be followed:

1. The sample was placed at least $50 \mathrm{~cm}$ distance from the thermal camera.

2. The room temperature was recorded.

3. IR source interferences were minimized. All windows were shielded to prevent any stray IR radiation.

4. A waiting period of $\cong 7$ to 10 min was allowed for thermal adaptation after every laser shot. The sample area was cooled slightly with a fan and monitored by the thermal camera.

5. The thermal imager system calibrated using an external black-body source is recommended to check for any drift in the temperature sensitivity setting. The threshold temperature of around 0.7 of its maximum temperature values shall also be calibrated, as it is a reference point that the thermal imagers use to differentiate an evaluated temperature from normal temperature.

\subsection{Image Processing}

The photothermal image is filtered from low-frequency thermal noise and high-frequency electrical noise and then integrated to produce values proportional to the thermal potential. Image segmentation and analysis are proposed as an efficient method for breast analysis using IR images. ${ }^{20-24}$ Different types of filters can be applied in order to remove the image artifacts and to optimize the signal-to-noise ratio by varying the used frequency band. For all images, three different types of filters with different parameters were applied, and the setting with the best image contrast and signal-to-noise ratio was chosen. Applying different filters with different parameters can change the image contrast.

For noise removal and to limit the background effect, a bandpass filter was applied after mapping the time domain into the frequency domain using Fourier transformation. The amplitude and temporal characteristics of the photoacoustic response depend upon the effective attenuation coefficient $\mu_{\text {eff }}$ of the tissue, which in turn is a function of the absorption coefficient $\mu_{a}$. Since the ratio of optical absorption in cancerous to normal tissues at $1064 \mathrm{~nm}$ is approximately a factor of 2 (Refs. 25- 27), there will be significant differences between the photoacoustic signatures of the two tissue types, thus providing a means of discriminating between normal and cancerous tissues. We can localize the tumor areas by calculating the photoacoustic response packs using edge-detection methods.

Edge detection is a well-developed field on its own within image processing. Region boundaries and edges are closely related, since there is often a sharp adjustment in intensity at the region boundaries. Edge-detection techniques are therefore used as the base of another segmentation technique..$^{20,21,28,29}$ The edges identified by edge detection are often disconnected. To segment an object from an image, however, one needs closed-region boundaries. Discontinuities are bridged, if the distance between the two edges is within 0.7 of its maximum temperature values.

We have designed a worksheet toolbox to extract the boundaries of the breasts, because it is one of the most precise edge-detection operators and has been widely used. . $2,23,28,29^{2}$ It is robust to noise and implements a Gaussian function to smooth the image and to obtain the magnitude and orientation of the gradient for each pixel.
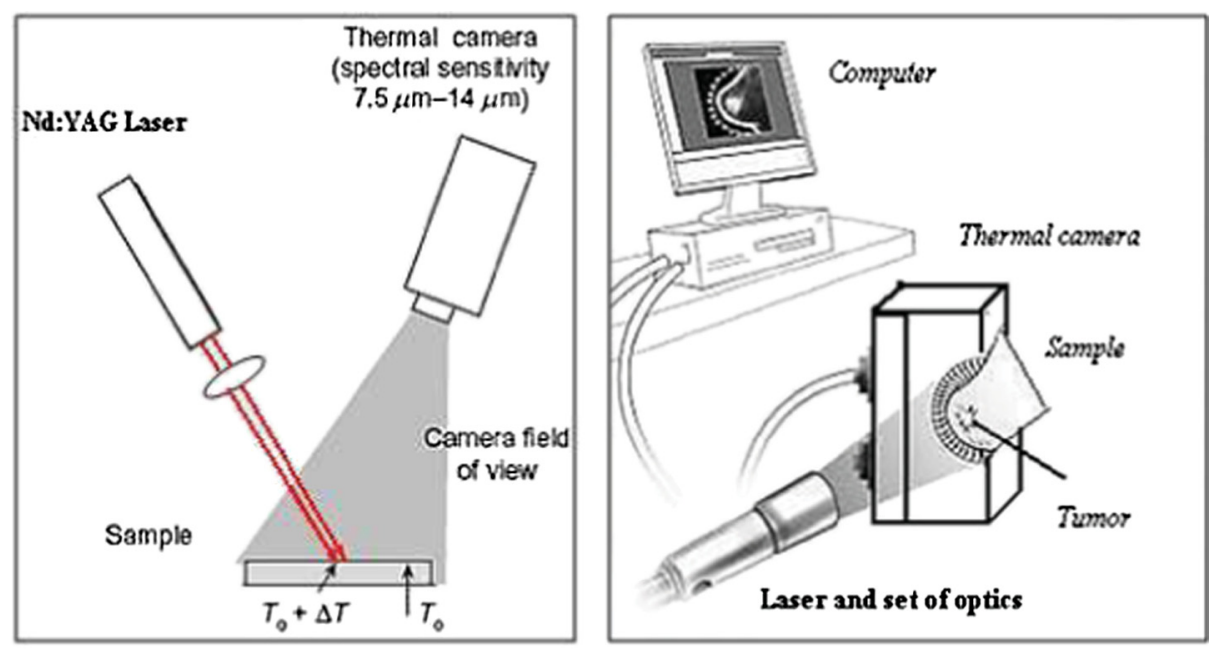

Fig. 2 Schematic of the photothermal imaging for the detection of human breast cancer. 


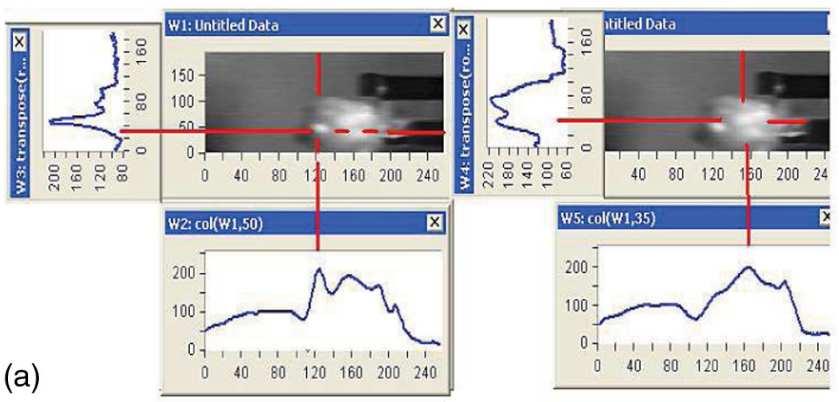

(b)

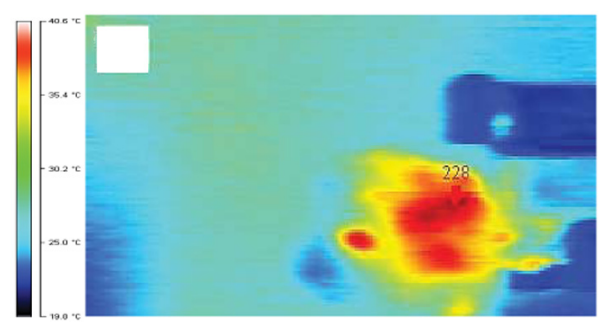

Fig. 3 In vivo near-infrared (NIR) imaging of a small tumor. (a) An NIR image taken with 5 -s radiation. High-tumor contrast is seen as the peak temperature intensity in gray scale. (b) A color photothermal image for the same sample (the red color represents the tumor position and its size).

\section{Results and Discussion}

Figures 3 and 4 show the photothermal images taken from the breast samples of a 55-year-old patient affected by breast cancer; the first sample taken from a controlled breast tumor of size $0.5 \mathrm{~cm}^{3}$ and the other sample taken from the affected tissue of size $2 \mathrm{~cm}^{3}$.

All samples of more than 15 biopsies (some of these irradiated sample are seen at end of this section in either of traditional gray or rainbow styles) were irradiated for $5 \mathrm{~s}$ at $0.6 \mathrm{~W} \mathrm{~cm}^{-2}$ with the 1064-nm NIR laser with a laser spot size of $4.4 \mathrm{~cm}^{2}$ (Fig. 2).

The laser power was kept constant throughout the irradiation. This led to a rapid temperature rise in the first minute, which then leveled to approximately 39 to $40^{\circ} \mathrm{C}$ over the next $5 \mathrm{~s}$.

The temperature was monitored continuously by thermal imaging and checked periodically by a thermoprobe placed directly in contact with the tumor. The physical appearance of the tumor was whitened. The tumors of the first sample had an average size of $\sim 22 \mathrm{~mm}^{3}$ in the start of irradiation. Thermal images, taken at various time points during the heating, showed that the tissue heated up very fast and reached an average temperature of $39^{\circ} \mathrm{C}$.

By contrast, the tumors reached temperatures of more than $40^{\circ} \mathrm{C}$ after $5 \mathrm{~s}$, while the laser spot size was much larger than the tumor $\left(4.4 \mathrm{~cm}^{2}\right.$ as compared with a tumor size of less
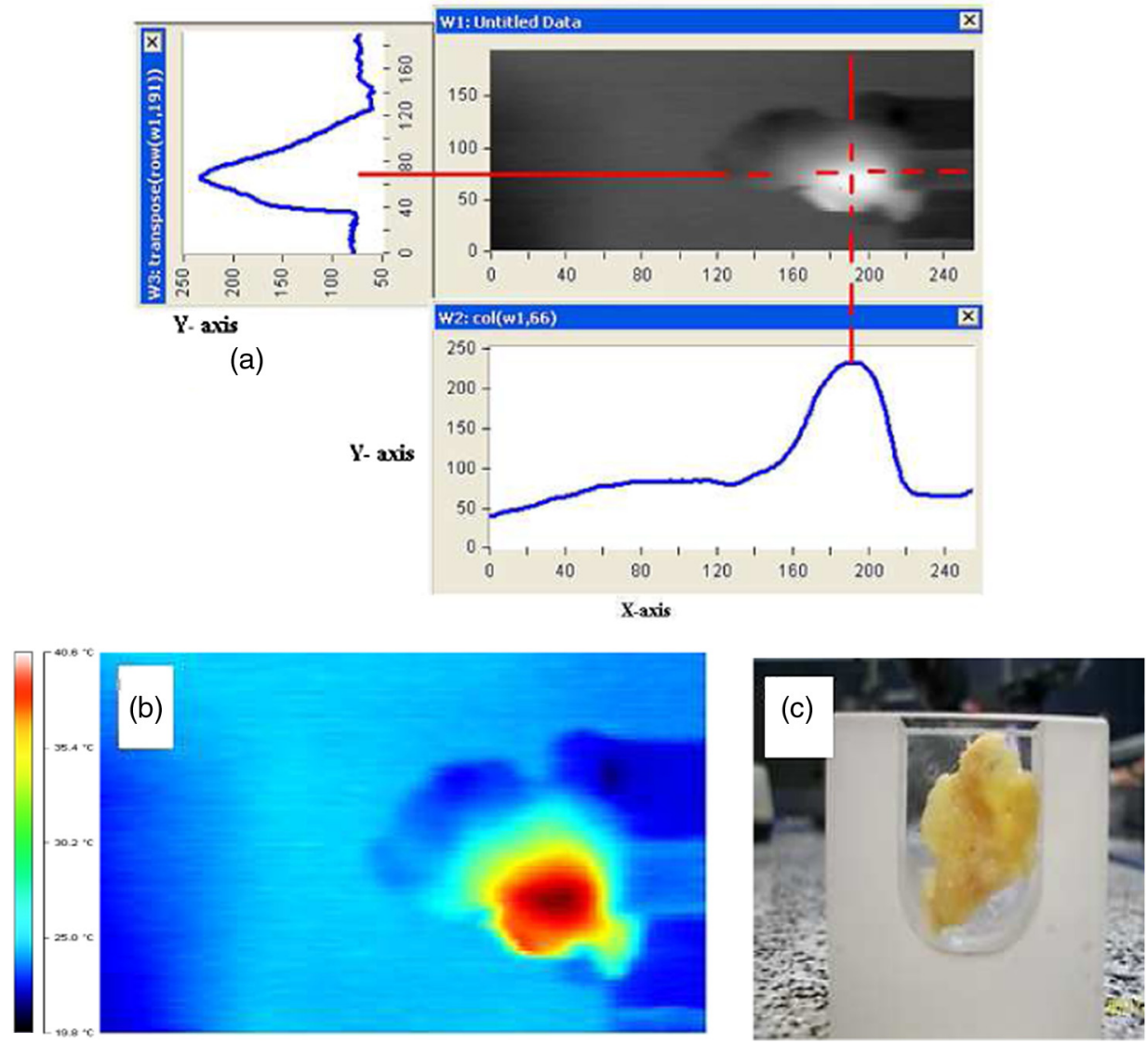

Fig. 4 In vivo NIR imaging of a large tumor. (a) A gray thermal image of a breast cancer-bearing tumor using NIR laser irradiation at a power of $2 \mathrm{~W} \mathrm{~cm}^{-2}$, (b) a color thermal image of the same sample, and (c) an optical image of the same sample. 

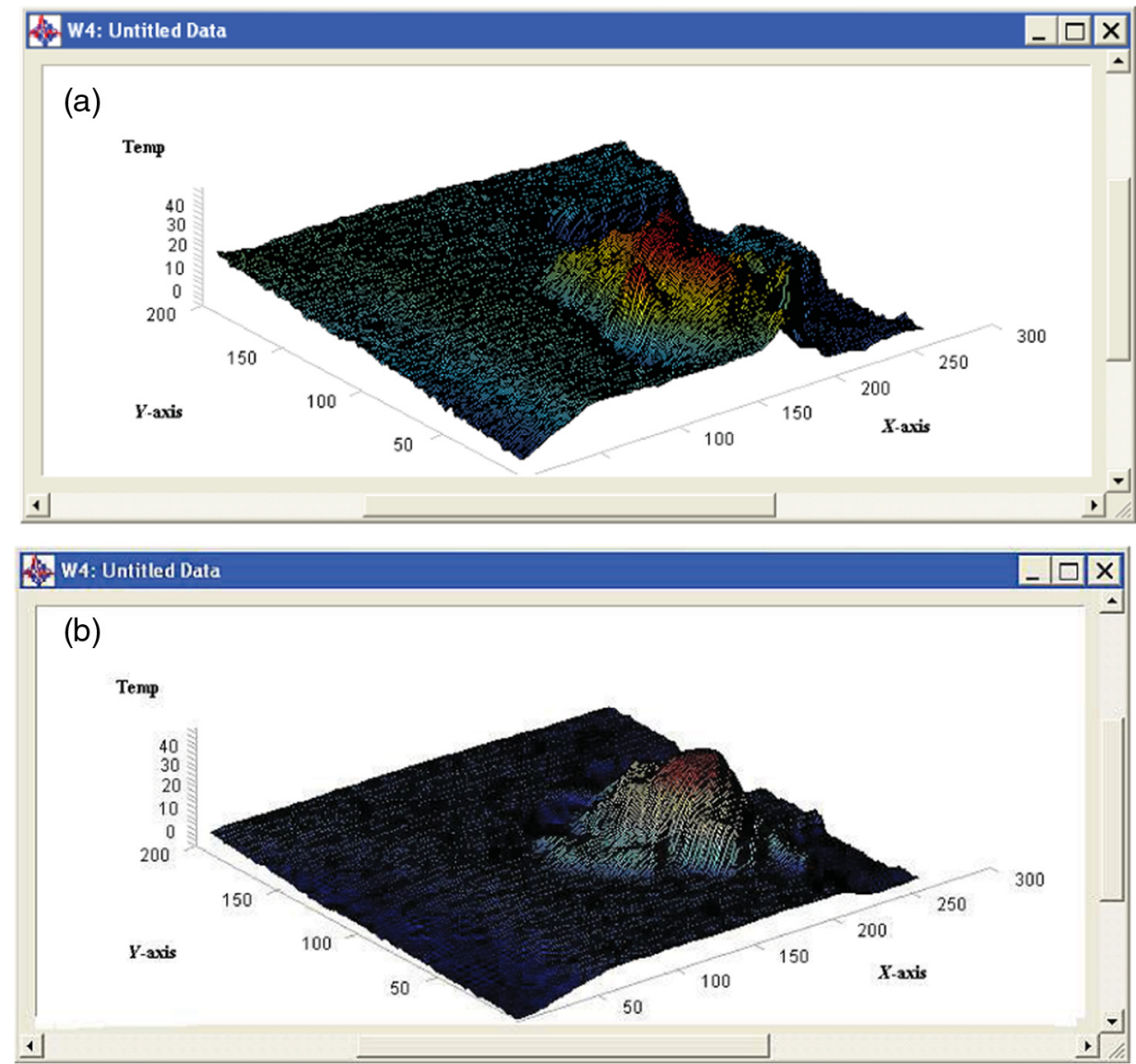

Fig. 5 Three-dimensional plot for (a) small tumor size $\left(<0.5 \mathrm{~cm}^{3}\right)$ and (b) large tumor size $\left(>0.5 \mathrm{~cm}^{3}\right)$.
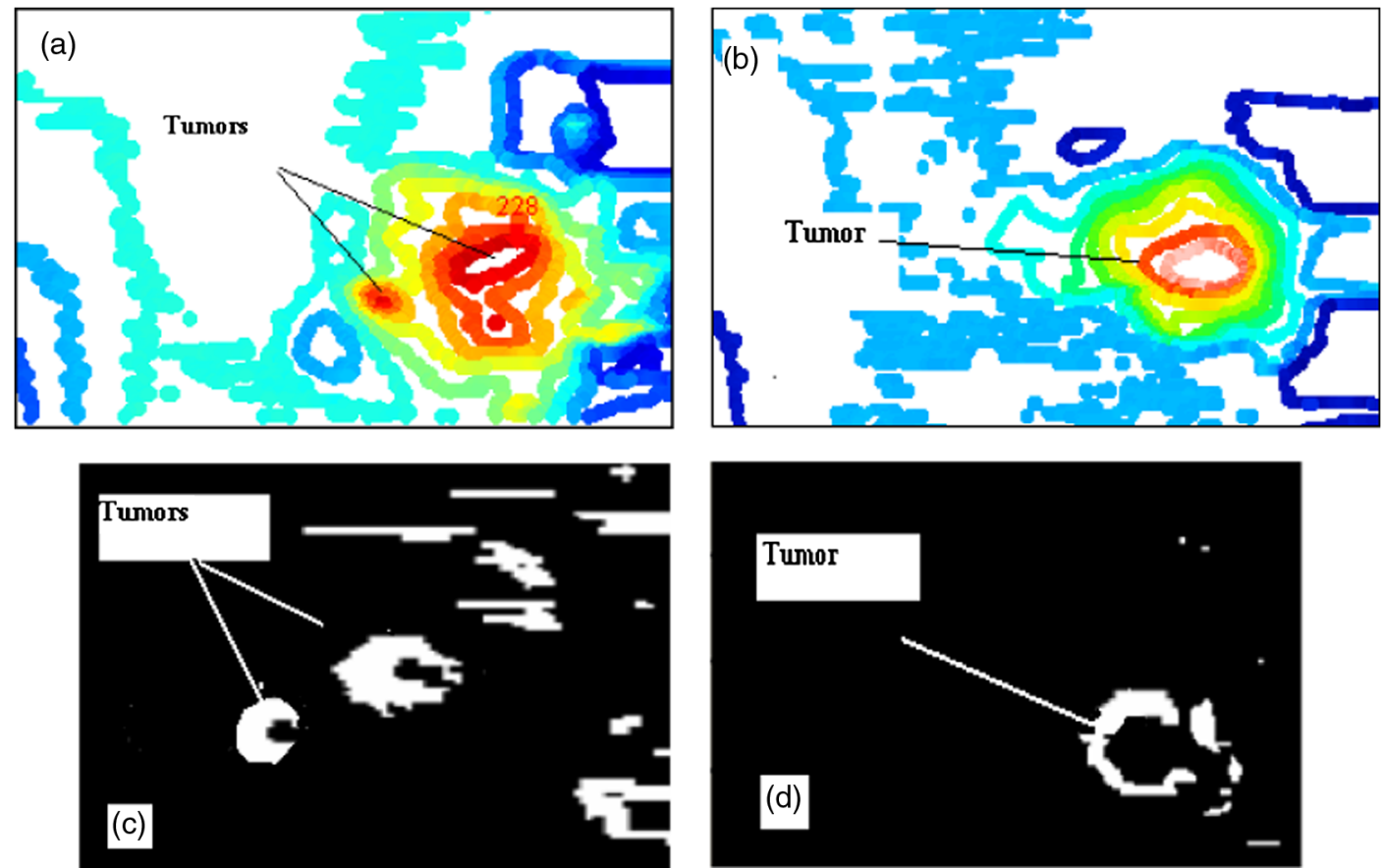

Fig. 6 Temperature contours after $1 \mathrm{~s}$ for (a) small tumor size $\left(<0.5 \mathrm{~cm}^{3}\right)$ and (b) large tumor size $\left(>0.5 \mathrm{~cm}^{3}\right)$. Edge detection for NIR imaging for (c) small tumor size $\left(<0.5 \mathrm{~cm}^{3}\right)$ and (d) large tumor size $\left(>0.5 \mathrm{~cm}^{3}\right)$. 

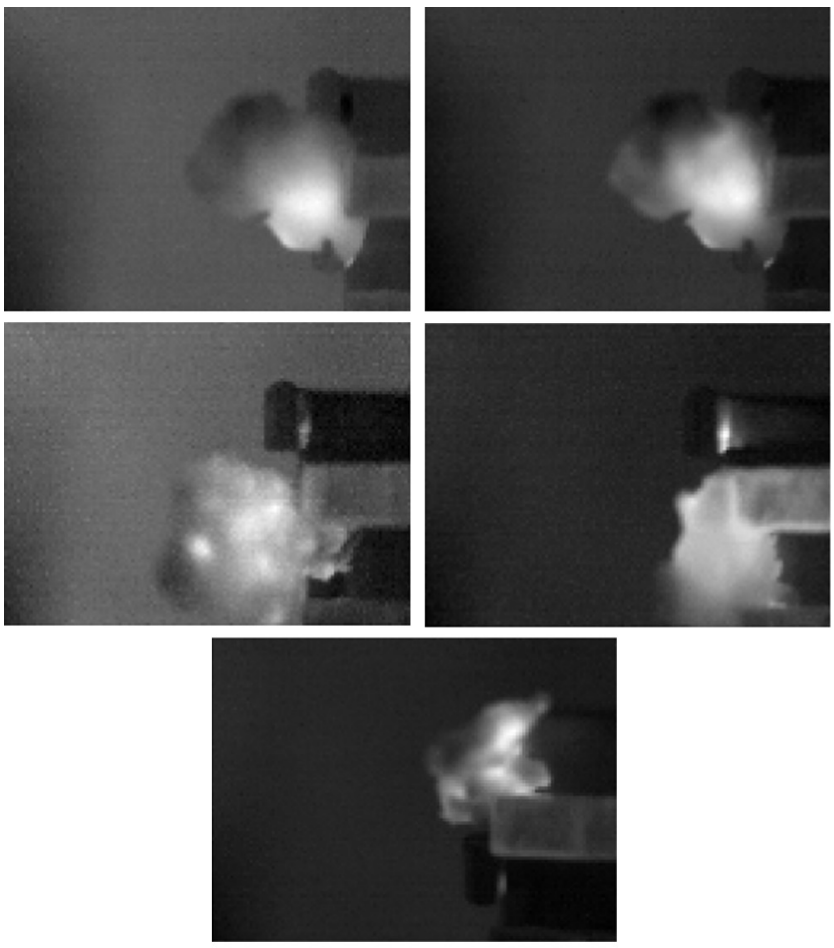

(a)
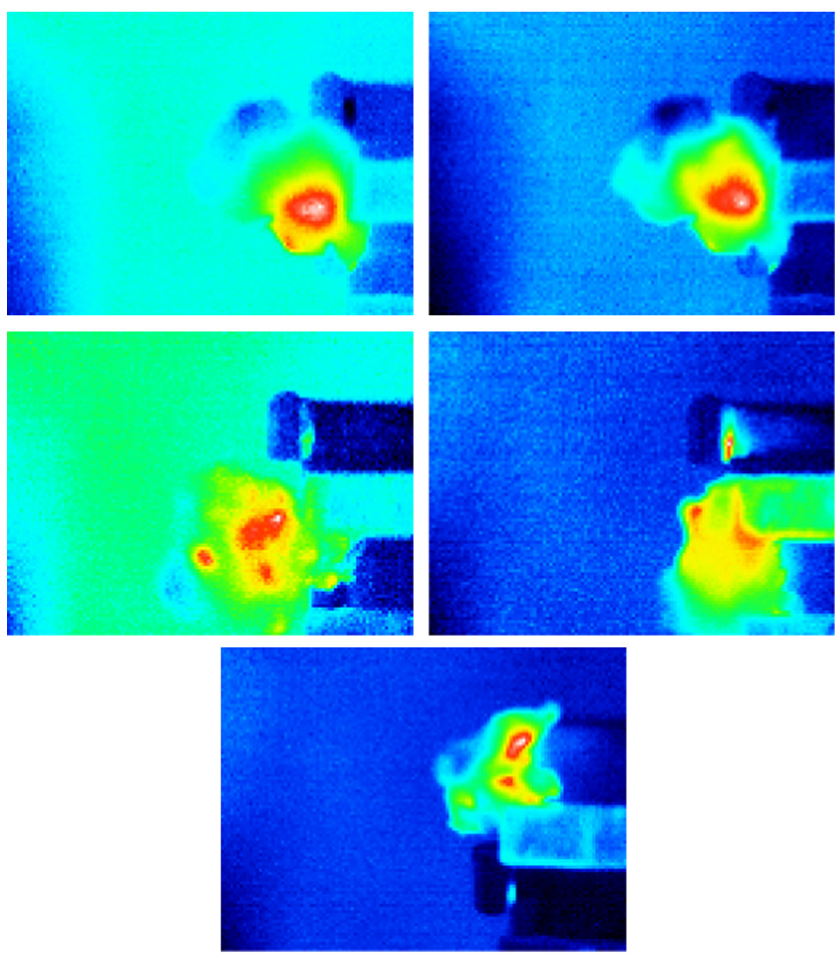

(b)

Fig. 7 (a) Thermal images of different breast tumor sizes and locations in gray format. (b) Thermal images of different breast tumor sizes and locations in color format.

than $0.5 \mathrm{~cm}^{2}$ ) and thermal imaging indicated that the healthy, illuminated tissue remained closer to normal body temperatures, as shown in Fig. 3. This confirms that the tumors were responsible for the absorption of laser light and subsequent heating of the tumor tissue. The second sample was considered "high risk" due to the large tumor size $>0.5 \mathrm{~cm}^{3}$ (Fig. 4). In strong contrast, for comparison, we examined the NIR photothermal heating capabilities of $1064 \mathrm{~nm}$. The absorption coefficient for breast tumor is higher than normal tissue..$^{30}$ The tumors heated at $2 \mathrm{~W} \mathrm{~cm}^{-2}$ formed an eschar and diminished. Based on thermal imaging, the high-intensity peaks, as shown in Figs. 5(a) and $5(\mathrm{~b})$, indicated the tumor position which matched with the physician report.

Photothermal imaging afforded high-passive accumulation in tumors relative to the surrounding organs and provided an opportunity to combine imaging and photothermal therapy of tumors by utilizing the intrinsic optical and thermal properties of cancerous cell. Also, the depth penetration of the NIR laser becomes an issue when treating tumors deep within the body. Since the penetration depth is less than $1 \mathrm{~cm}$ at this wavelength, techniques other than an external laser are necessary to effectively treat deep body tumors. For example, in some cases, the laser source can be fiber coupled and threaded into the body. When the NIR photothermal images show a high signal in the tumor and a low signal in the surrounding tissue, we are able to heat the tumors without incurring damage to healthy tissue. The features of the heat patterns may include the statistical parameters such as skewness, variation, and kurtosis. The larger the tumor size, the steeper the temperature variation. ${ }^{31}$ How the temperature varies in the heat pattern is also important. Two heat patterns with the same temperature range, as shown in Figs. 6(a) and 6(b), one may be steeper than the other for the same area, have the different temperature variations or the same temperature variation occurs in the different areas. The cumulative histogram which describes the temperature variation with an area can reflect the asymmetry. The asymmetry can be measured by the bilateral ratio of the quantitative features including skewness, variation, kurtosis, temperature difference $(\Delta T)$, the maximum ratio between temperature difference, and area $(\Delta T / S)$.

Figures 6(c) and 6(d) illustrate the tumors position using stable edge-detection techniques; from these results, we can use it with photothermal therapy and tumor treatments. We select a biopsy at different tumor sizes and locations from women at almost the same edge and same tumor degree (nearly 55 years, first degree), image it using our experiment, and save the thermal image in gray and color formats, as shown in Figs. 7(a) and 7(b), to calculate the accuracy of our system and the ability to detect a tumor. As shown in the figures, the system has a power tool for detecting the tumor location with high degree of accuracy and matching with the physician report.

\section{Discussions and Conclusions}

Photothermal laser heating of tumors coupled with the ability to image and visualize heat intake into tumors makes photothermal imaging a promising potential method for future photothermal diagnosis and treatment. The novelty of the current work lies in several areas as mentioned in this scenario; first, on the imaging front, we have been able to achieve high-resolution photothermal imaging of tumor with size less than $2 \mathrm{~cm}^{3}$ using NIR laser. The ability to track the tumor distribution using edge detection is also an important feature for the diagnosis of breast in early stage cancer and in the monitoring processes. Finally, we took advantage of the high-optical absorbance of breast cancer in the NIR band to heat the tumor volume to the point of complete cell death, which may be used as a treatment process. More than 15 cancerous biopsies are investigated using the same approach and were in good agreement with traditional clinical measurements 
with more precision in extracting the exact size and shape of the cancerous cells. ${ }^{15-18,20-28}$ Also, by using this technique with the help of advanced digital image processing, we would be able to distinguish and differentiate between different grade levels of tumor and cancerous cells. The overall photothermal imaging system is a powerful tool for real-time breast cancer detection with high resolution and contrast, which enables us to detect tumors at the size of millimeters in diameter compared with the photoacoustic imaging system. ${ }^{32}$ This photothermal imaging system is more simple and compact and provides real-time noninvasive monitoring and imaging. In comparison, off-time photoacoustic imaging requires an array of piezoeletric transducer to acquire acoustic signals at multiple layers and special digital signal processing software. ${ }^{32}$

\section{References}

1. Z. S. Deng and J. Liu, "Analytical study of bioheat transfer problems with spatial or transient heating on skin surface or inside biological bodies," J. Biomech. Eng. 124, 638-649 (2002).

2. A. Katzir, Lasers and Optical Fibers in Medicine, Elsevier Science \& Technology Books, Academic Press, New York (1993).

3. R. W. Waynant, Lasers in Medicine, Taylor \& Francis (2010).

4. G. S. Terentyuk et al., "Laser-induced tissue hyperthermia mediated by gold nanoparticles: toward cancer phototherapy," J. Biomed. Opt. 14(2), 021016 (2009).

5. G. Von Maltzahn et al., "Computationally guided photothermal tumor therapy using long-circulating gold nanorod antennas," Cancer Res. 69(9), 3892-3900 (2009).

6. W. J. Yang, Bio-Thermal Fluid Sciences: Principles and Application, Hemisphere, Washington, DC (1989).

7. J. C. Chen and Y. C. Huang, "Thermocapillary flow of surface melting due to a moving heat flux," Int. J. Heat Mass Transfer 34(3), 663-671 (1991).

8. J. J. Crochet et al., "Temperature distribution in selective laser-tissue interaction," J. Biomed. Opt. 11(3), 034031 (2006).

9. W. R. Chen et al., "Chromophore-enhanced in vivo tumor cell destruction using an 808-nm diode laser," Cancer Lett. 94(2), 125-131 (1995).

10. R. A. London et al., "Laser-tissue interaction modeling with LATIS," Appl. Opt. 36, 9068-9074 (1997).

11. W. F. Cheong, S. A. Prahl, and A. J. Welch, "A review of the optical properties of biological tissues," IEEE J. Quantum Electron. 26(12), 2166-2185 (1990).

12. M. H. Niemz, Laser-Tissue Interactions; Fundamentals and Applications, Springer-Verlag, Berlin, Germany (1996).

13. A. Katzir, Lasers and Optical Fibres in Medicine, Chapter 4, Academic Press, San Diego, California (1993).

14. R. W. Waynant, Lasers in Medicine, CRC Press, Boca Raton, Florida (2002).

15. L. Boquete et al., "Automated detection of breast cancer in thermal infrared images, based on independent component analysis," J. Med. Syst. 36, 103-111 (2012).

16. A. M. Ovechkin and G. Yoon, "Infrared imaging for screening breast cancer metastasis based on abnormal temperature distribution," J. Opt. Soc. Korea 9, 157-161 (2005).

17. H. Qi and N. A. Diakides, "Thermal infrared imaging in early breast cancer detection- A survey of recent research," in Proc. of the 25th Annual International Conference of the IEEE Engineering in
Medicine and Biology Society (EMBS), Cancun, Mexico, Vol. 2, pp. 1109-1112 (2003).

18. L. Fass, "Imaging and cancer: a review," Mol. Oncol. 2, 115-152 (2008).

19. A. F. El-Sherif and Y. El-Sharkawy, "Laser-induced photothermal technique used for detection of caries in human tooth," Proc. SPIE 6843, 68430B (2008).

20. L. S. S. Reddy et al., "A novel image segmentation technique for detection of breast cancer," Int. J. Inf. Technol. Knowl. Manage. 2(2), 201-204 (2010).

21. N. M. Basheer and M. H. Mohamme, "Segmentation of breast masses in digital mammograms using adaptive median filtering and texture analysis," Int. J. Recent Technol. Eng. (IJRTE) 2(1), 2277-3878 (2013).

22. X. Yan et al., "The application of the edge sharpening operator to the breast near-infrared imaging," Wuhan Univ. J. Nat. Sci. 7(4), 421-425 (2002).

23. L. Xiang-ji, D. Run-tao, and C. Jing, "Order-statistical edge enhancing filters for noisy images," J. Tianjin University 32(6), 687-690 (1999).

24. R. Qiu-yi, Digital Image Processing Technique, Electronics Industry Press, Beijing (2001).

25. P. Wang, J. R. Rajian, and J.-X. Cheng, "Spectroscopic imaging of deep tissue through photoacoustic detection of molecular vibration," J. Phys. Chem. Lett. 4, 2177-2185 (2013).

26. R. A. Bitar Carter et al., "FT-Raman spectroscopy study of human breast tissue," Proc. SPIE 5321, 190-197 (2004).

27. M. Ali Ansari, M. Erfanzadeh, and E. Mohajerani, "Mechanisms of laser-tissue interaction: II. Tissue thermal properties," J. Lasers Med. Sci. 4(3), 99-106 (2013).

28. I. K. Maitra, S. Nag, and S. K. Bandyopadhyay, "Accurate breast contour detection algorithms in digital mammogram," Int. J. Comput. Appl. 25(5), 0975-8887 (2011).

29. N. Singh et al., "GUI based automatic breast cancer mass and calcification detection in mammogram images using K-means and fuzzy C-means methods," Int. J. Mach. Learn. Comput. 2(1), 7-12 (2012).

30. S. Xie, H. Li, and B. Li, "Recent progress in medical photonics," Sci. China Ser. G-Phys. Mech. Astron. 52(6), 856-863 (2009).

31. F. Xu, T. J. Lu, and K. A. Seffen, "Biothermomechanical behavior of skin tissue," Acta. Mech. Sin. 24, 1-23 (2008).

32. W. Xia et al., "Design and evaluation of a laboratory prototype system for 3D photoacoustic full breast tomography," Biomed. Opt. Express 4(11), 2555-2569 (2013).

Yasser H. El-Sharkawy received his $\mathrm{Ms}$ degree and $\mathrm{PhD}$ degree in Biomedical engineering from MTC College in 1998 and 2003 respectively. His PhD work concerned the breast cancer detection using photoacoustic imaging. Currently, he is associated professor and head of bio-photonics group at biomedical engineering department, MTC, Cairo, Egypt.

Ashraf F. El-Sherif has over 8 years' experience in diode-pumped solid-state and fiber laser research and development. He served as a post-doctoral researcher at CREOL, College of Optics \& Photonics, University of Central Florida, Orlando in 2012-2013, and as a research scientist at LPL, Laser Townes Institute, CREOL, developing high power Tm-doped photonic crystal fiber lasers with application in backside silicon machining and $\mathrm{Yb}$ :fiber laser systems, respectively, primarily for materials processing applications. He has achieved his $\mathrm{PhD}$ in laser photonics and modern optics, from Laser Photonics Research Group, University of Manchester, UK, in 2003. He has achieved an associate professor title in October 2009, and he is a lecturer and faculty member within the Engineering Physics Department, MTC, Cairo, Egypt. 